\title{
Ciclo de vida de Triatoma dimidiata Latreille, 1811 (Hemiptera, Reduviidae) en condiciones de laboratorio: producción de ninfas para ensayos biológicos
}

\author{
Marlene Reyes ${ }^{1}$, Víctor Manuel Angulo² \\ 1 Escuela de Biología, Facultad de Ciencias, Universidad Industrial de Santander, Bucaramanga, Colombia \\ 2 Centro de Investigaciones en Enfermedades Tropicales, CINTROP, Universidad Industrial de Santander, \\ Piedecuesta, Colombia
}

Introducción. A pesar de la importancia de Triatoma dimidiata como vector de la enfermedad de Chagas, poco se conoce de su ciclo biológico y de la producción eficiente de insectos disponibles para ensayos biológicos.

Objetivo. Determinar las características del ciclo de vida en el laboratorio y establecer las condiciones del estado nutricional para la producción eficiente de ninfas de $V$ estadio para ensayos biológicos.

Materiales y métodos. Se determinaron los tiempos de desarrollo de los estadios de ninfa en condiciones controladas de laboratorio hasta alcanzar la fase adulta. Se llevó a cabo una cría masiva de ninfas de $\mathrm{V}$ estadio, alimentadas y pesadas después de diferentes periodos de ayuno, distribuidas en rangos de peso para obtener la mayor proporción de individuos.

Resultados. El tiempo medio de paso de huevo a adulto fue de 269 días, con un amplio rango de duración (174 a 598 días) y, para los estadios I, II, II, IV y V, fue de 33, 37, 41, 61 y 69 días, respectivamente, con una mortalidad de $22 \%$. Se obtuvo una eficiencia de $76 \%$ en ninfas de $\mathrm{V}$ estadio, alimentadas después de 22 días de ayuno, en el rango de 201 a $300 \mathrm{mg}$ de peso.

Conclusión. T. dimidiata presentó un tiempo de desarrollo intermedio entre los triatominos con amplio rango para algunos individuos, posiblemente debido a la irregularidad en su alimentación. La identificación de un rango de peso homogéneo después de 22 días de ayuno con gran producción de ninfas de $\mathrm{V}$ estadio, facilita la aplicación de protocolos estandarizados para establecer criterios de selección de compuestos insecticidas utilizables en los programas de control.

Palabras clave: Triatominae, triatoma, etapas del ciclo de vida, enfermedad de Chagas, Colombia.

Life cycle of Triatoma dimidiata Latreille, 1811 (Hemiptera, Reduviidae) under laboratory conditions: production of nymphs for biological tests

Introduction. Despite the importance of Triatoma dimidiata as a vector of Chagas disease, little is known of its life cycle and the efficient production of these insects for biological tests.

Objective. Life cycle characteristics in the laboratory were described and optimum nutritional conditions were established for the efficient production of nymphs $\mathrm{V}$ stage for biological tests.

Materials and methods. We determined the time of development of the nymphal stage under controlled laboratory conditions until reaching the adult stage. In a massive rearing of stage $\mathrm{V}$ nymphs, fed and weighted after varying periods of fasting, distributed in weight ranges to obtain the largest proportion of individuals.

Results. The time mean from egg to adult was 269 days, with a wide range of duration (174 to 598 days) and the times required for 1 st, 2nd, 3rd, 4th and 5th stage development was 33, 37, 41, 61 and 69 days, respectively, with a mortality of $22 \%$. The optimum treatment was 22 days of fasting, in which $76 \%$ of the nymphs reached stage $V$ with a weight range from 201 to $300 \mathrm{mg}$.

Conclusion. Triatoma dimidiata presented development time with broad range for some individuals, possibly due to the irregularity in the food availability. A homogenous weight range was attained with a regime of 22 days of fasting with an optimum production of stage $\mathrm{V}$ nymphs. 
Production of similarly sized insects facilitate the application of standardized protocols to establish criteria for selecting compounds insecticides used in control programs.

Key words: Triatominae, triatoma, life cycle stages, Chagas disease, Colombia.

La enfermedad de Chagas causada por Trypanosoma cruzi y transmitida a los humanos por diferentes especies de insectos triatominos que ocupan hábitats domiciliarios, afecta entre 16 y 18 millones de personas, y 100 millones están en riesgo de infección (1).

En Centroamérica y la región andina, la reducción delos niveles de infestación por Rhodniusprolixus, debida a la iniciación de programas estatales de intervención, ha hecho que Triatoma dimidiata emerja como la especie de mayor importancia en la transmisión en algunas zonas geográficas de varios países como Colombia, donde se ha visto una amplia y creciente diseminación en los últimos años (2-4).

Su presencia en diferentes hábitats, dentro $y$ alrededor de viviendas rurales y urbanas, y su carácter eurifágico, que incluye al hombre como huésped, lo implican como un vector importante en la transmisión de la infección por T. cruzi (5-8). Este comportamiento y la menor eficacia de los insecticidas utilizados, han provocado que los programas de intervención no logren los mismos resultados obtenidos en zonas infestadas con Triatoma infestans y $R$. prolixus (9).

A pesar de su importancia en salud pública, sólo unos pocos estudios sobre su ciclo de vida en Centroamérica han aportado información sobre las características de desarrollo de los estados de ninfa en el laboratorio (10) y no existe información sobre estas características en cepas de Suramérica.

La manipulación de condiciones, como temperatura, humedad, fuentes alimentarias y periodos de alimentación, sobre una especie, permiten

\section{Correspondencia:}

Marlene Reyes, Laboratorio de Entomología, Centro de Investigaciones en Enfermedades Tropicales, Universidad Industrial de Santander, Sede UIS Guatiguará, km 2 Piedecuesta, Santander, Colombia.

Tel: (576) 634 4000; ext 3526; fax: (576) 6550808 marejez1@ hotmail.com

Recibido:19/05/08; aceptado:27/11/08 conocer sobre sus ciclos de desarrollo, dinámica de población y capacidad de colonización, conocimientos necesarios para el mantenimiento de colonias que serán objeto de estudios de evaluación de la actividad insecticida.

Los insecticidas y sus formulaciones, usados para el control de los vectores de la enfermedad de Chagas, han sido evaluados directamente en campo, sin previos ensayos biológicos de laboratorio bajo condiciones estandarizadas. Por esta razón, el Programa de Investigación y Adiestramiento en Enfermedades tropicales (Tropical Disease Research) de la Organización Mundial de la Salud (OMS), adoptó un protocolo estandarizado de ensayos biológicos para cuantificar, a nivel de laboratorio y de campo, la eficacia y la efectividad de sus principios activos y sus formulaciones. La expectativa fallida del uso en campo del DDT en las campañas iniciales de control de triatominos, ha demostrado la importancia de estos ensayos, a fin de prever y manejar eventuales fracasos futuros de los tratamientos de control (11).

Entrabajospreviossehan encontrado importantes diferencias en la susceptibilidad a insecticidas entre triatominos de diferente especie, estadio, edad en el mismo estadio, periodo de ayuno, proximidad a la muda y estado nutricional (1217); algunas de estas condiciones básicas se han determinado para especies vectoras de la enfermedad de Chagas, como $T$. infestans y $R$. prolixus $(18,19)$.

La mayor susceptibilidad encontrada en ninfas alimentadas de estadio II de $T$. infestans y en ninfas de estadio $V$ de $T$. dimidiata, que sugieren una mayor absorción de los insecticidas en esta condición nutricional $(14,20)$, nos motiva a desarrollar estrategias que permitan la producción eficiente de ninfas alimentadas, con pesos homogéneos para los ensayos biológicos.

A pesar de que algunos autores consideran que $T$. dimidiata es una especie inadecuada para el uso en laboratorio, por el prolongado tiempo 
de desarrollo (21) y de que no hay estudios sobre la determinación de las condiciones requeridas para la producción eficiente y la utilización de estadios juveniles de $T$. dimidiata en la evaluación de estrategias de control, dada su importancia epidemiológica, se hace necesario, y es el propósito de este estudio, conocer las características del ciclo de vida y establecer las condiciones de producción eficiente para obtener ninfas disponibles en número suficiente para los ensayos biológicos en la evaluación de insecticidas.

\section{Materiales y métodos}

\section{Material biológico}

Se utilizó una cepa de $T$. dimidiata procedente de una vivienda rural del municipio de San Joaquín, área endémica para la enfermedad de Chagas en el departamento de Santander, colonizada en el laboratorio tres años atrás y mantenida sin aporte de material externo, en condiciones ambientales constantes de laboratorio a $25 \pm 2{ }^{\circ} \mathrm{C}$, $70 \%$ a $80 \%$ de humedad relativa, fotoperíodo de 12:12 y alimentándolas cada 15 días con sangre de gallina.

Ciclo de vida de T. dimidiata en laboratorio: a partir de una cohorte de 102 huevos mantenidos en las condiciones ya señaladas, seguidos hasta su eclosión, seestablecieron el tiempoy la proporción de eclosión y se determinó el tiempo medio de incubación y el porcentaje de viabilidad.

Las ninfas eclosionadas se colocaron en lotes de 20 individuos en frascos plásticos de $4 \mathrm{x}$ por $10 \mathrm{~cm}$, de boca ancha cubierta con un tul y alimentadas cada 15 días con sangre de gallina.

Se registró cada día el número de ninfas mudadas y el número de individuos muertos, y el tiempo en días entre cada muda para cada individuo. Con estos registros se estableció el tiempo medio de desarrollo para cada estadio, el tiempo promedio de huevo a adulto y el porcentaje de mortalidad por estadio y total.

\section{Producción y estandarización del peso en diferentes tiempos de ayuno en ninfas de estadio V de T. dimidiata}

Se colocaron diez parejas de hembras y machos en un recipiente para lograr la cópula y la postura; los huevos obtenidos diariamente se trasladaron a otro recipiente hasta su eclosión. Las ninfas emergidas fueron llevadas a otros recipientes en lotes de 30 ninfas y alimentadas cada 15 días.

Se llevó a cabo una revisión diaria de cada recipiente para seleccionar las ninfas que realizaron la muda al siguiente estadio; éstas se transfirieron sucesivamente a otros recipientes, hasta conseguir ninfas del cuarto estadio; las que alcanzaron el quinto estadio durante la semana de revisión, se separaron en frascos rotulados con la fecha de la semana de muda.

Entre uno y cuatro de estos lotes de ninfas se sometieron a diferentes tiempos de ayuno ( 7 , $8,11,12,13,15$ y 22 días); fueron alimentados luego durante una hora y pesados en una balanza analítica, siete días después de esta primera ingestión; también se pesaron los lotes de ninfas sin alimentar, con 10 a 12 días de ayuno.

El peso de cada individuo de cada lote se registró en una base de datos y, de acuerdo con este registro, los individuos se clasificaron en ocho rangos según su peso, con intervalos de $100 \mathrm{mg}$. Este proceso requirió un tiempo aproximado de 15 meses.

\section{Análisis de los resultados}

Ciclo de vida: para el cálculo de la duración promedio del ciclo biológico, se estableció la media de duración en días para cada estadio de desarrollo, de la siguiente manera: suma del total de días de duración en el estadio de ninfa de cada uno de los individuos (incluyendo los individuos de menor y de mayor duración)/número de individuos en el estadio; el índice de mortalidad se estableció con la siguiente fórmula: número de individuos muertos en cada estadio de ninfa/número de individuos que iniciaron en el estadio x 100 .

Producción y estandarización del peso y tiempo de ayuno en ninfas de estadio $V$ de $T$. dimidiata: para determinar los rangos de pesos con mayores proporciones de ninfas de estadio $V$ en relación con los días de ayuno, se estableció el porcentaje de individuos obtenidos en cada rango de peso según los días de ayuno, en las condiciones nutricionales (alimentadas y sin alimentar). 


\section{Resultados}

\section{Ciclo de vida de T. dimidiata en laboratorio}

Se observó la eclosión de la totalidad de los huevos y se determinó un índice de viabilidad del $100 \%$, un periodo medio de incubación de 27,9 días, con un mínimo y un máximo de 24 a 30 días, respectivamente. En el desarrollo de los estadios de ninfa de $\mathrm{NI}$ a NV, se observó un amplio rango de duración: desde un mínimo de 17 días en individuos que pasaron de NI a NII, hasta un máximo de 142 días en individuos que pasaron de NIV a NV. Las ninfas de primero y segundo estadio, en promedio, completaron su desarrollo en 33 y 38 días, respectivamente; las ninfas de tercero y cuarto estadio lo completaron en 41 y 61 días en promedio; el quinto estadio fue el más prolongado, con una duración de 69 días para llegar al estado adulto. Ochenta ninfas completaron su desarrollo hasta el estado adulto (48 machos y 32 hembras). El tiempo medio de desarrollo de huevo a adulto fue de 269 días, con un mínimo de 174 días y un máximo de 598 (cuadro 1).

El proceso de alimentación no fue uniforme, ni obedeció en todos los individuos al cronograma programado de ofertas alimentarias; algunos no se alimentaban fácilmente, ni a total repleción, con continuas interrupciones, ingerían cantidades variables de sangre y su tiempo de ingestión se prolongaba hasta más de una hora; otros nunca ingirieron sangre aunque trataban de hacerlo. En el primer estadio el crecimiento fue más uniforme en relación con el resto de los estadios inmaduros. El índice de mortalidad global de la cohorte fue de $22 \%$; en los estadios juveniles fue bajo, $3 \%$ para las N5 y entre $4 \%$ y $6 \%$ para los estadios de NI a N4.

Producción y estandarización del peso y tiempo de ayuno en ninfas de estadio $V$ de $T$. dimidiata

Se obtuvieron 1.918 ninfas en 25 lotes conformados según los días de ayuno. Se observó una proporción de ninfas alimentadas obtenidas en los rangos de peso entre 201 y $300 \mathrm{mg}$ por encima del $35 \%$, después de cualquier periodo de ayuno, que variaron entre $35,7 \%$, a los ocho días de ayuno, y $76 \%$, después de 22 días de ayuno, cuando se obtuvieron 120 ejemplares; ésta fue la más alta. Aunque algunos lotes sometidos a siete días de ayuno produjeron el mayor número de ninfas para los rangos de peso de 201 a 300 $\mathrm{mg}$ y de $101 \mathrm{a} 200 \mathrm{mg}$, su rendimiento fue menor, ya que se requirió una gran cantidad de ninfas. En las ninfas sin alimentar sólo se obtuvieron dos rangos de peso: 0,01 a 100,00 mg, con una proporción de $88 \%$, y 101 a $200 \mathrm{mg}$, con una proporción de $12 \%$ (cuadro 2 ).

\section{Discusión}

La importancia de $T$. dimidiata como vector de $T$. cruzi en Colombia y en Centroamérica ha motivado, desde la década de los 50, estudios relacionados con el conocimiento de su biología y la características del ciclo de desarrollo en el laboratorio.

\section{Ciclo de vida}

El tiempo medio de desarrollo bajo las condiciones de laboratorio (nueve meses) en este estudio,

Cuadro 1. Ciclo de desarrollo de huevo-adulto de Triatoma dimidiata expresado en días. Media y desviación estándar (media \pm DE).

\begin{tabular}{lccccc}
\hline \multirow{2}{*}{ Estadio } & $\mathbf{n}$ & \multicolumn{3}{c}{ Duración en días } & $\begin{array}{c}\% \\
\text { Mortalidad }\end{array}$ \\
\cline { 3 - 5 } & & Mínimo & Máximo & Media \pm DE & 0 \\
Huevo a NI & 102 & 24 & 30 & $27,9 \pm 2,0$ & 6 \\
NI-NII & 96 & 17 & 105 & $33,0 \pm 15,5$ & 4 \\
NII-NIII & 92 & 22 & 128 & $37,6 \pm 14,1$ & 5 \\
NIII-NIV & 87 & 30 & 104 & $41,0 \pm 10,6$ & 4 \\
NIV-NV & 83 & 30 & 89 & $60,7 \pm 21,4$ & 3 \\
NV-AD & 80 & 51 & 598 & $68,5 \pm 8,4$ & 22 \\
Huevo-adulto & 80 & 174 & & 268,91 & \\
\hline
\end{tabular}


Cuadro 2. Proporción de ninfas de $\mathrm{V}$ estadio de Triatoma dimidiata, sin alimentar y alimentadas después de diferentes días de ayuno, obtenida por rangos de peso.

\begin{tabular}{|c|c|c|c|c|c|c|c|c|}
\hline \multirow{3}{*}{$\begin{array}{l}\text { Rango } \\
\text { Pesos } \\
\text { N V T. dimidiata }\end{array}$} & \multirow{3}{*}{$\begin{array}{c}\text { Ninfas V } \\
\text { sin alimentar } \\
\text { 10-12 días } \\
n\end{array}$} & \multicolumn{7}{|c|}{ Días de ayuno prealimentación } \\
\hline & & 7 & 8 & 11 & 12 & 13 & 15 & 22 \\
\hline & & $\begin{array}{c}n \\
(\%)\end{array}$ & $\begin{array}{c}n \\
(\%)\end{array}$ & $\begin{array}{c}n \\
(\%)\end{array}$ & $\begin{array}{c}n \\
(\%)\end{array}$ & $\begin{array}{c}n \\
(\%)\end{array}$ & $\begin{array}{c}n \\
(\%)\end{array}$ & $(\%)$ \\
\hline $0,0001<x<=100,00$ & 69 & $\begin{array}{c}20 \\
(2,25)\end{array}$ & $\begin{array}{c}4 \\
(3,6)\end{array}$ & $\begin{array}{c}0 \\
0(0)\end{array}$ & $\begin{array}{c}1 \\
(1,7)\end{array}$ & $\begin{array}{c}7 \\
(1,8)\end{array}$ & $\begin{array}{c}0 \\
(0)\end{array}$ & $\begin{array}{c}1 \\
(0,6)\end{array}$ \\
\hline $101,00<x<=200,00$ & 9 & $\begin{array}{c}253 \\
(28,5)\end{array}$ & $\begin{array}{c}50 \\
(44,6)\end{array}$ & $\begin{array}{c}13 \\
(31,7)\end{array}$ & $\begin{array}{c}1 \\
(1,7)\end{array}$ & $\begin{array}{c}147 \\
(38,1)\end{array}$ & $\begin{array}{c}55 \\
(28,2)\end{array}$ & $\begin{array}{c}28 \\
(17,7)\end{array}$ \\
\hline $201,00<x<=300,00$ & 0 & $\begin{array}{c}350 \\
(39,4)\end{array}$ & $\begin{array}{c}40 \\
(35,7)\end{array}$ & $\begin{array}{c}20 \\
(48,7)\end{array}$ & $\begin{array}{c}30 \\
(50,8)\end{array}$ & $\begin{array}{l}189 \\
(50)\end{array}$ & $\begin{array}{c}91 \\
(46,6)\end{array}$ & $\begin{array}{l}120 \\
(76)\end{array}$ \\
\hline $301,00<x<=400,00$ & 0 & $\begin{array}{c}158 \\
(17,8)\end{array}$ & $\begin{array}{c}14 \\
(12,5)\end{array}$ & $\begin{array}{c}5 \\
(12,2)\end{array}$ & $\begin{array}{c}26 \\
(44)\end{array}$ & $\begin{array}{c}36 \\
(9,3)\end{array}$ & $\begin{array}{c}42 \\
(21,5)\end{array}$ & $\begin{array}{c}8 \\
(5)\end{array}$ \\
\hline $401,00<x<=500,00$ & 0 & $\begin{array}{c}70 \\
(7,8)\end{array}$ & $\begin{array}{c}3 \\
(2,7)\end{array}$ & $\begin{array}{c}3 \\
3(7,3)\end{array}$ & $\begin{array}{c}1 \\
(11,8)\end{array}$ & $\begin{array}{c}6 \\
(1,6)\end{array}$ & $\begin{array}{c}5 \\
(2,5)\end{array}$ & $\begin{array}{c}1 \\
(0,6)\end{array}$ \\
\hline $501,00<x<=600,00$ & 0 & $\begin{array}{c}31 \\
(3,5)\end{array}$ & $\begin{array}{c}0 \\
(0)\end{array}$ & $\begin{array}{c}0 \\
(0)\end{array}$ & $\begin{array}{c}0 \\
(0)\end{array}$ & $\begin{array}{c}1 \\
(0,26)\end{array}$ & $\begin{array}{c}2 \\
(1)\end{array}$ & $\begin{array}{c}0 \\
(0)\end{array}$ \\
\hline $601,00<x<=700,00$ & 0 & $\begin{array}{c}6 \\
(0,67)\end{array}$ & $\begin{array}{c}1 \\
(0,9)\end{array}$ & $\begin{array}{c}0 \\
(0)\end{array}$ & $\begin{array}{c}0 \\
(0)\end{array}$ & $\begin{array}{c}0 \\
(0)\end{array}$ & $\begin{array}{c}0 \\
(0)\end{array}$ & $\begin{array}{c}0 \\
(0)\end{array}$ \\
\hline $701,00<x<=1.000,0$ & & $\begin{array}{c}1 \\
(0,11)\end{array}$ & $\begin{array}{c}0 \\
(0)\end{array}$ & $\begin{array}{c}0 \\
(0)\end{array}$ & $\begin{array}{c}0 \\
(0)\end{array}$ & $\begin{array}{c}0 \\
(0)\end{array}$ & $\begin{array}{c}0 \\
(0)\end{array}$ & $\begin{array}{c}0 \\
(0)\end{array}$ \\
\hline TOTAL & 78 & 888 & 112 & 41 & 59 & 386 & 195 & 158 \\
\hline
\end{tabular}

fue más largo que el observado por Otálora para una cepa colombiana (6,5 a 7 meses) (22) y estuvo dentro del rango descrito por Hernández Mora para otra cepa colombiana (23), pero fue más corto que el establecido por Zeledón (10 a 11 meses) en una cepa centroamericana en condiciones de laboratorio y con un tiempo de colonización semejante al utilizado en este estudio (10).

Fue comparable con el de Triatoma sordida, Triatoma patagonica y Triatoma pallidipennis $(6$ a 11 meses) $(24,25)$, pero mayor que el reportado para otras especies del mismo género, como $T$. infestans (4,7 meses), Triatoma maculata (4,2 a 6 meses) y Triatoma mazzottii (7 meses) (26-29), de igual forma que para especies de otros géneros, como $R$. prolixus (3 a 5 meses) y $R$. colombiensis $(4,81$ meses) $(24,30)$. Se ha observado que, en el género Triatoma, el ciclo biológico que más tiempo requiere es el de Triatoma lenti (12 meses) (31). Estas observaciones sobre el desarrollo y el mantenimiento de una cepa autóctona de $T$. dimidiata en condiciones de laboratorio, aportan información reciente sobre las características de su ciclo biológico, para Colombia.

\section{Hipótesis de variaciones entre ciclos de $T$. dimidiata}

Las diferencias observadas en los rangos y en el tiempo medio de duración del ciclo de vida de la cepa colombiana utilizada en este estudio con respecto otras cepas de Centroamérica y Colombia, pueden deberse a las condiciones previas al ensayo, como el tiempo de colonización en el laboratorio; a variaciones de las condiciones del laboratorio en el ensayo, o a diferencias de taxonomía numérica (phenetics) y genéticas de la población ya demostradas para esta especie (32-34).

Las dificultades en la alimentación de un buen número de individuos pueden explicar la prolongación del tiempo de desarrollo. T. dimidiata ha sido considerada por varios investigadores una especie poco agresiva, en comparación con otras de gran importancia epidemiológica, como $T$. infestans y $R$. prolixus, debido a su capacidad de resistencia al ayuno (35). Esto parece ser un mecanismo compensatorio. Los sistemas neurosecretores responden a la señal activadora de un alimento sanguíneo y por ausencia de 
este estímulo, ante la falta de total repleción, se afecta la producción de la hormona estimuladora de la ecdisis (ecdicsona) y, por ende, el tiempo de desarrollo de la ninfa $(36,37)$.

Esta característica, la gran viabilidad de los huevos y la baja mortalidad reveladas en este estudio, en comparación con especies como $R$. prolixus y $T$. infestans, y la gran fecundidad y fertilidad de las hembras observada en Centroamérica $(35,38,39)$, pueden representar una importante ventaja biológica para la supervivencia de las colonias y explicar su éxito en la competencia en su ambiente natural.

Su carácter "eurífago" le permite aprovechar cualquier tipo de huésped (silvestre, doméstico) y prosperar en diferentes hábitats, incluyendo ecótopos artificiales (7-9,40-43); pero, además, puede explicar la persistencia de poblaciones extradomiciliarias como fuente de infestación de las viviendas, después de la utilización de insecticidas (40).

\section{Producción y estandarización del peso y tiempo de ayuno en ninfas de estadio $V$ de $T$. dimidiata}

El estudio demuestra que es factible la producción masiva de estadios inmaduros de esta especie en el laboratorio. Sin embargo, es necesario considerar el prolongado tiempo de desarrollo ( 6 a 11 meses) que implica el cuidado por parte de un auxiliar y las condiciones necesarias para su mantenimiento en el laboratorio.

El hallazgo de un rango de peso homogéneo entre 201 y $300 \mathrm{mg}$ después de 22 días de ayuno, con la mayor eficiencia en la producción masiva de ninfas de estadio $\mathrm{V}$, disponibles para su utilización en ensayos biológicos, representa una buena perspectiva en la determinación de parámetros esenciales para la aplicación de protocolos estandarizados en el seguimiento de la susceptibilidad y la resistencia a insecticidas utilizados comúnmente sin evaluación previa.

Ahora es posible establecer como soporte a los programas gubernamentales de control en Latinoamérica, un extensivo programa de vigilancia de la resistencia a los insecticidas de esta especie de importancia epidemiológica.

\section{Agradecimientos}

Los autores agradecen las sugerencias aportadas por los dos evaluadores anónimos, las cuales mejoraron el manuscrito original.

\section{Conflicto de intereses}

No hay conflicto de intereses.

\section{Financiación}

Este estudio fue financiado por UNDP/World Bank/WHO Special Programme for Research and Training in Tropical Diseases (TDR), proyecto ID: 990163.

\section{Referencias}

1. Moncayo A. Chagas disease: Current epidemiological trends after the interruption of vectorial and transfusiona transmission in the Southern Cone Countries. Mem Inst Oswaldo Cruz. 2003;98:577-91.

2. Guhl F, Angulo V, Restrepo M, Nicholls S, Montoya R. Estado del arte de la enfermedad de Chagas en Colombia y estrategias de control. Biomédica. 2003;23:31-4.

3. Molina JA, Gualdrón LE, Brochero LH, Olano VA, Barrios D, Guhl F. Distribución actual e importancia epidemiológica de las especies de triatominos (Reduviidae: Triatominae) en Colombia. Biomédica. 2000;20:344-60.

4. Guhl F, Vallejo GA. Interruption of Chagas disease transmission in the Andean countries: Colombia. Mem Inst Oswaldo Cruz. 1999;94:413-5.

5. Christensen AH, Sousa EO, Vasquez AM. Host feeding profiles of Triatoma dimidiata in peridomestic habitats of Western Panama. Am J Trop Med Hyg. 1988;38:477-9.

6. Calderón AO, Chinchilla M, García F, Vargas M. Preferencias alimentarias de Triatoma dimidiata (Hemiptera: Reduviidae) procedente de la meseta central de Costa Rica a finales del siglo XX. Parasitología al Día. 2001;25:3-4.

7. Zeledón R, Solano G, Zúñiga A, Swartzwelder JC. Biology and ethology of Triatoma dimidiata (Latreille, 1811) II. Habitats and blood sources. J Med Entomol. 1973;10:363-70.

8. Zeledón R, Calvo N, Montenegro V, Seixas LE, Arévalo C. A survey on Triatoma dimidiata in an urban area of the province of Heredia, Costa Rica Rio de Janeiro. Mem Inst Oswaldo Cruz. 2005;100:607-12.

9. Angulo VM. Comportamiento de Triatoma dimidiata: Un reto para su control. Biomédica. 2005;25:80-3.

10. Zeledón R, Guardia VM, Zúñiga A, Swartzwelder JC. Biology and ethology of Triatoma dimidiata (Latreille, 1811). I: Life cycle, amount of blood ingested, resistance 
to starvation and size of adults. J Med Entomol. 1970;7:313-9.

11. World Health Organization. Control of Chagas disease. Second report of the WHO Expert Committee. Technical report 2002. Series 905. Geneva: WHO; 2002. p. 39-40.

12. Fontán A, Zerba EN. Influence of the nutritional state of Triatoma infestans over the insecticidal activity of DDT. Comp Biochem Physiol C. 1992;101:589-91.

13. Rojas A, Lehane MJ, Schofield CJ, Fournet A. Comparative evaluation of pyrethroid insecticide formulations against Triatoma infestans (Klug): Residual efficacy on four substrates. Mem Inst Oswaldo Cruz. 2003;98:975-80

14. Reyes M, Angulo VM, Sandoval CM. Efecto tóxico de ß-cipermetrina, deltametrina y fenitrotión en cepas de Triatoma dimidiata (Latreille, 1811) y Triatoma maculata (Erichson, 1848) (Hemiptera, Reduviidae). Biomédica. 2007;27(Suppl.1):75-82.

15. Oliveira Filho AM. Differences of susceptibility of five triatomine species to pyrethroid insecticides: implications for Chagas' disease vector control. Mem Inst Oswaldo Cruz.1999;94:425-8.

16. Wood E, Picollo MI, Zerba E. Comparación entre la variación de la capacidad detoxificante y la diferente susceptibilidad al insecticida malatión entre ninfas $\mathrm{V}$ de distinta edad de Triatoma infestans. Rev Asoc Med Arg.1993;81:153-62.

17. Fontán A, Zerba E. Mode of entry of insecticides in Triatoma infestans. Arch Insect Biochem Physiol. 1987;43:13-323.

18. Nelson MJ. Experiencias en el monitoreo de niveles de susceptibilidad de los triatominos a los insecticidas en las Américas. Acta Toxicol Arg. 1994;2:29-58.

19. World Health Organization. Protocolo de evaluación de efecto insecticida sobre triatominos. Acta Tóxico Arg. 1994;2:29-32.

20. Fontán A, Zerba EN. Influence of the nutritional state of Triatoma infestans over the insecticidal activity of DDT. Comp Biochem Physiol C. 1992;101:589-91.

21. Paz RR. Ciclo de vida y datos biométricos de Triatoma Longipennis(Usinger)(Hemiptera:Reduviidae,Triatominae) (tesis). Ciudad de México: Universidad Nacional Autónoma de México; 1996. p. 96.

22. Otálora B. Triatoma dimidiata (Latreille). Anal Soc Biol Bogotá. 1952;5:135-7.

23. Hernández MC. Infección natural Triatoma capitata Usinger, 1939 por el Trypanosoma cruzi. Rev Fac Med Unal. 1947;15:465-76.

24. Canale MD, Jurberg J, Carcavallo UR, Galvao C, Mena SC, Silva RD, et al. Bionomics of some species. In: Carcavallo UR, Galindez GI, Jurberg J, Lent H, editores. Atlas of Chagas disease vectors in the Américas. Volumen III. Río de Janeiro: Fiocruz-Inc; 1999. p. 839-90.
25. Martínez JA, Gala KD. Biology of Triatoma pallidipennis Stal 1945 (Hemiptera: Reduviidae: Triatominae) under laboratory conditions. Mem Inst Oswaldo Cruz. 1999;94:837-9.

26. Rabinovich JE. Vital statistics of Triatominae (Hemiptera: Reduviidae) under laboratory conditions. I. Triatoma infestans Klug. J Med Entomol. 1972;9:351-70.

27. Moura JF, Vargas A, Almeida E, Esperança G, Souza R, Ramos E, et al. A Triatoma maculata (Hemiptera, Reduviidae, Triatominae) population from Roraima, Amazon region, Brazil, has some bionomic characteristics of a potential Chagas' disease vector. Rev Inst Med Trop Sao Paulo. 2005;47:131-7.

28. Feliciangeli MD, Rabinovich JE. Vital statistics of Triatominae (Hemiptera: Reduviidae) under laboratory condition. J Med Entomol.1985;22:43-8.

29. Malo EA, Ramírez RA, Cruz L, Rojas J. Lyfe cycle and influence of age and feeding on the first mating of Triatoma mazoottii (Hemiptera:Reduviidae) Mem Inst Oswaldo Cruz. 1993;88:203-6.

30. Arévalo A, Carranza J, Guhl F, Clavijo J, Vallejo G. Comparación del ciclo de vida de Rhodnius colombiensis Moreno, (Jurberg \& Galvao,1999) y Rhodnius prolixus (Stal,1872) (Hemiptera, Reduviidae, Triatominae) en condiciones de laboratorio. Biomédica. 2007;27(Suppl.1):119-29.

31. Carcavallo UR, Mena SC,Canale MD. Ciclo de vida de Triatoma lenti Sherlock, Serafim,1967 (Hemiptera, Reduviidae, Triatominae). Entomología y Vectores. 1994;1:43-9.

32. Dorn P, Monrroy C, Curtis A. Triatoma dimidiata (Latreille, 1811): A review of its diversity across its geographic range and the relationship among populations. Infect Genet Evol. 2007;7:343-52.

33. Bargues M, Klisiowicz D, Gonzalez F, Ramsey J, Monroy C, Ponce C, et al. Phylogeography and genetic variation of Triatoma dimidiata, the main Chagas' disease vector in Central America, and its position within the genus triatoma. PLoS Negl Trop Dis. 2008;2:1-19.

34. Arroyo CM, Esteban L, Catalá S, Angulo VM. Variación del fenotipo antenal de poblaciones del domicilio, peridomicilio y silvestres de Triatoma dimidiata (Hemiptera: Reduviidae) en Santander, Colombia. Biomédica. 2007;27(Suppl.1):92-100.

35. Zeledón R. El Triatoma dimidiata (Latreille,1811) y su relación con la enfermedad de Chagas. San José: Editorial EUNED; 1981. p .146.

36. Friend WG, Smith JJ. Fisiología de los triatominos con especial referencia a la alimentación por sangre. En: Carcavallo RU, Rabinovich JE, Tonn RJ, editores. Factores biológicos y ecológicos en la enfermedad de Chagas. Tomo I. Epidemiología-vectores. Washington: OPS/OMS; 1985. p. 55-81. 
37. Zeledón R, Alvarado R, Jirón LF. Observations on the feeding and defecation patterns of three triatomine species (Hemiptera: Reduviidae) Acta trop. 1977;34:6577.

38. Canale D,Carcavallo RU. Triatoma infestans (Klug). En: Carcavallo RU, Rabinovich JE, Tonn RJ, editores. Factores biológicos y ecológicos en la enfermedad de Chagas. Tomo I. Epidemiología -vectores. Washington, D.C.: OPS/OMS; 1985. p. 237-50.

39. Carcavallo RU, Tonn R. Rhodnius prolixus (Stal). En: Carcavallo RU, Rabinovich JE, Tonn RJ, editores. Factores biológicos y ecológicos en la enfermedad de Chagas. Tomo I. Epidemiología -vectores. Washington, D.C.: OPS/OMS; 1985. p. 209-17.

40. Angulo VM. Ensayo de estrategias de control y vigilancia de Triatoma dimidiata en Colombia. En: Guhl $F$, editor. Primer Taller Internacional sobre Control de la Enfermedad de Chagas; Curso de Diagnóstico, Manejo y Tratamiento de la Enfermedad de Chagas; VI Reunión de la Iniciativa Andina para el Control de la Enfermedad de Chagas. Bogotá, D.C.: Ediciones Uniandes; 2005. p. 91-102.

41. Zeledón AR, Guardia VM, Zúñiga A, Swartzwelder JC. Biology and ethology of Triatoma dimidiata (Latreille, 1811) II. Life span of adults and fecundity and fertility of females. J Med Entomol.1970;7:462-9.

42. Zeledón R. Triatoma dimidiata (Latreille, 1811). En: Carcavallo RU, Rabinovich JE, Tonn RJ, editores. Factores biológicos y ecológicos en la enfermedad de Chagas. Tomo I. Epidemiología-vectores. Washington, D.C.: OPS/OMS; 1985. p. 225-36.

43. Zeledón R, Montenegro VM, Zeledón O. Evidence of colonization of man-made ecotopes by Triatoma dimidiata (Latreille,1811) in Costa Rica. Mem Inst Oswaldo Cruz. 2001;96:659-60. 